\title{
Capacidad de infección de suspensiones de Pyricularia oryzae con diferentes periodos de conservación
}

\section{Suspensions infectivity of Pyricularia oryzae with different periods of conservation}

\begin{abstract}
Oscar Frutos ${ }^{1}$, Yessica Reyes ${ }^{2}{ }^{\bullet}$, Alice Chávez ${ }^{1,2}{ }^{\oplus}$, Mohan Kohli ${ }^{2}{ }^{1}$ Universidad Católica "Nuestra Señora de la Asunción”. Asunción, Paraguay. ${ }^{2}$ Cámara Paraguaya de Exportadores y Comercializadores de Cereales y Oleaginosas. Asunción, Paraguay. *Autor de correspondencia: magalizrc@ gmail.com.
\end{abstract}

Frutos, O., Reyes, A., Chávez, A. \& Kohli, M. (2021). Capacidad de infección de suspensiones Pyricularia oryzae con diferentes periodos de conservación. Investigaciones y Estudios-UNA, 12(2), 310. https://doi.org/10.47133/IEUNA2121b

Resumen. El brusone del trigo causado por Pyricularia oryzae patotipo Triticum, es una de las mayores amenazas para la producción de trigo en Sudamérica (5-100\% de perdidas). Una de las características aún desconocidas del patógeno es el periodo por el cual se pueden conservar suspensiones de inóculo fúngico. Conocer este aspecto permitiría planificar ensayos para búsqueda de genotipos resistentes con gran número de líneas. El objetivo del trabajo fue determinar la infectividad de suspensiones de Pyricularia oryzae con diferentes periodos de conservación $(0,8,15$, 24 y 30 días) en dos variedades de trigo (Caninde 1 y Caninde 11), en condiciones de campo e invernadero. Se utilizó el diseño completamente al azar con 5 tratamientos y 10 repeticiones en ambos ambientes, se evaluó el tipo de reacción de hojas y espigas mediante escalas, los datos fueron analizados mediante la prueba de Kruskall-Wallis. Se observó que a mayor tiempo de conservación del inoculo la capacidad de infección de las conidias de $P$. oryzae, disminuye en ambas variedades. Las suspensiones con cero días de conservación infectaron con mayor índice a ambas variedades en los dos periodos de evaluación. Relacionando las medias de infección y la cantidad de esporas sin germinar en las suspensiones con distintos periodos de conservación, en la variedad Caninde 1, el coeficiente de correlación fue de 0,98 , mientras que en la variedad Caninde 11 fue 0,97; estos valores indican una fuerte correlación entre las variables, pues a medida que aumenta la cantidad de conidios sin germinar, aumenta la capacidad de infección de las suspensiones. Las variedades Caninde $1 \mathrm{y}$ Caninde 11, mostraron una reacción resistente y susceptible, respectivamente, frente a la cepa P014038 de Pyricularia oryzae.

Palabras clave. brusone, conservación de inóculo, resistencia genética, trigo. 
Abstract. Wheat Blast, caused by Pyricularia oryzae patotype Triticum, is one of the greatest threats to wheat production in South America (5$100 \%$ losses). One of the still unknown characteristics of the pathogen is the period for which inoculum suspensions can be kept. Knowing this aspect would allow planning tests to search for resistant genotypes with a large number of lines. The objective of the work was to determine the infectivity of Pyricularia oryzae suspensions with different conservation periods $(0,8,15,24$ and 30 days) in two varieties of wheat (Caninde 1 and Caninde 11), under field and greenhouse conditions. It was observed that the longer the inoculum conservation time, the infection capacity of $P$. oryzae conidia decreases in both varieties. Suspensions with zero days of conservation infected both varieties with a higher rate in the two evaluation periods. Relating the infection means and the amount of ungerminated spores in the suspensions with different conservation periods, in variety Caninde 1, the correlation coefficient was 0.98, while in variety Caninde 11 it was 0.97 ; These values indicate a strong correlation between the variables, since as the number of ungerminated conidia increases, the infection capacity of the suspensions increases. The Caninde 1 and Caninde 11 varieties showed a resistant and susceptible reaction, respectively, against the Pyricularia oryzae strain P014-038.

Keywords. brusone, inoculum conservation, genetic resistance, wheat.

\section{INTRODUCCIÓN}

En la zafra 2019/2020 en Paraguay, el área de siembra fue de 400.000 ha, la producción comercial alcanzó las 750.000 toneladas y el rendimiento fue de $1875 \mathrm{~kg}$. ha ${ }^{-1}$, ese mismo año se logró exportar toneladas. El 99\% de lo exportado fue destinado a Brasil y el $1 \%$ a Bolivia y Uruguay (CAPECO, 2021). Si bien se logra una buena producción en el país, esta se ve amenazada año tras año por varios factores, como el clima, plagas que aparecen con mayor intensidad y enfermedades, siendo una de las principales amenazas para la producción de trigo en el país la enfermedad denominada Pyricularia o brusone.

El brusone del trigo causado por Pyricularia oryzae patotipo Triticum, es uno de los más serios problemas para la producción de trigo en Sudamérica, cuyo principal síntoma es la presencia de espigas con la porción superior al punto de infección blanca y la inferior verde, las pérdidas producidas son del $5-100 \%$ de la producción, esto se ha convertido en una amenaza a la expansión de los campos de trigo en las regiones tropicales y sub tropicales, en nuestro país esta enfermedad se manifiesta de forma endémica (Kohli et al., 2011).

Dada la importancia de esta enfermedad en nuestro país, es necesario investigar aspectos del patógeno que permitan ayudar al Programa Nacional de Mejoramiento de Trigo en la identificación y selección de genotipos resistentes a la enfermedad.

Uno de los aspectos desconocidos del patógeno, es el periodo por el cual se pueden conservar suspensiones de inóculo. Conocer este aspecto, permitiría planificar ensayos con gran número de 
líneas a campo, permitiendo testear mayor número de genotipos por año.

El objetivo de este trabajo fue determinar la infectividad de suspensiones de Pyricularia oryzae con diferentes periodos de conservación en dos variedades de trigo, Caninde 11 y Caninde 1 en condiciones de campo e invernadero.

\section{MATERIALES Y MÉTODOS}

El trabajo se realizó en el Centro de Investigación Hernando Bertoni, dependencia del Instituto Paraguayo de Tecnología Agraria con sede en la ciudad de Caacupé. Fue seleccionada la cepa monospórica P014-038, aislada de la variedad CD 150 durante el ciclo 2014, en el Campo Experimental Yhovy, Departamento de Canindeyú, perteneciente a la colección de hongos del proyecto Pyricularia en trigo del IPTACAPECO. La colección cuenta actualmente con más de 120 aislados monospóricos conservados por las técnicas de papel de filtro y PDA deshidratado a $-20^{\circ} \mathrm{C}$ en doble copia. La misma fue activada en medio de cultivo solido a base de Papa-Dextrosa-Agar a $25^{\circ} \mathrm{C}$ por 7 días. Posteriormente se procedió a la incubación por 10 días a en medio solido a base de Avena-DextrosaAgar $25^{\circ} \mathrm{C}$. Para estimular la esporulación el microorganismo fue sometido a estrés físico y mantenido en cámara húmeda $(90$ - 95\%) con luz constante a una temperatura de $25-28^{\circ} \mathrm{C}$ por 72 horas (Marangoni et al., 2013).

Tanto en campo como en invernadero, el diseño experimental utilizado fue completamente al azar con 5 tratamientos y 10 repeticiones. El experimento se realizó una sola vez. Los tratamientos consistieron en la inoculación de las variedades de trigo Caninde 11 y Caninde 1, estas variedades han sido reportadas como respectivamente, susceptibles y resistentes al fitopatógeno (Chávez \& Mohan-Kohli, 2018), con suspensiones de conidios de Pyricularia oryzae, elaboradas a partir de placas con cultivo puro del hongo y crioconservadas a $10^{\circ} \mathrm{C}$ durante cinco periodos de tiempo; T1: suspensión elaborada en el día (0 días de conservación), T2: 8 días de conservación, T3: 15 días de conservación, T4: 24 días de conservación, T5: 30 días de conservación. Las inoculaciones fueron efectuadas con una suspensión de 50.000 conidios $/ \mathrm{mL}$ de solución en los dos ambientes evaluados.

La inoculación en campo se realizó asperjando 10 espigas por tratamiento, utilizando un atomizador manual, cada espiga fue considerada una repetición. Inmediatamente luego de la aspersión, cada espiga fue cubierta con una funda de plástico, para mantener la humedad y favorecer la infección, durante 16 horas. Transcurrido ese tiempo, las fundas fueron retiradas (Chávez et al., 2020). La evaluación se realizó a los 15 y 30 días luego de la inoculación, respectivamente, utilizando la escala propuesta por Tagle et al. (2014). En donde se clasifica el tipo de infección siendo $0=\sin$ infección, $1=$ lesiones pequeñas, 1,5 $\mathrm{mm}, 2=$ lesiones de tamaño intermedio, $3 \mathrm{~mm}, 3=$ mezcla de glumas verdes y blancas, sin necrosis aparente, causado por una reacción de hipersensibilidad y $4=$ espiga completamente necrosada.

La inoculación en invernadero se realizó una vez que el trigo tuvo 3 a 4 hojas verdaderas, aproximadamente 1 mes luego de la siembra, 
asperjando 10 platines de cada variedad con cada suspensión, utilizando un aspersor manual. Inmediatamente luego se llevaron a una cámara húmeda, donde se le dieron las condiciones favorables para la infección, temperatura de $28^{\circ} \mathrm{C}$ y humedad relativa del $80 \pm 10 \%$, durante las 24 horas siguientes a la inoculación.

La evaluación de infección se realizó a los 15 días luego de la inoculación, se evaluó la cuarta hoja de cada planta utilizando la escala propuesta en hojas adaptada de Valent et al. (1991), la cual clasifica los síntomas en: $0=\operatorname{Sin}$ infección, $1=$ Lesiones puntuales del tamaño de una cabeza de alfiler de color marrón, 2= Lesiones elípticas 0 estriadas de $5 \mathrm{~mm}$ o más sin centro distinguible, $3=$ Lesiones redondeadas con borde oscuro y centro ceniza, en algunos casos con halo amarillo, 4= Lesiones típicas del brusone, elípticas con borde oscuro y centro ceniza, 5= Grandes lesiones elípticas con centro gris oscuro.

Para el análisis de los datos obtenidos a partir de las inoculaciones, se calcularon las medias de infección de cada tratamiento, debido a que los datos provenían del uso de escalas, se utilizó la prueba de Kruskall-Wallis, la cual compara medianas para establecer diferencias entre los tratamientos, utilizando el programa estadístico INFOSTAT.

Antes de cada inoculación, se evaluó el número de conidios sin germinar de cada suspensión una vez finalizado el periodo de conservación, mediante el conteo de los mismos con cámara de Neubauer siguiendo la metodología de French y Hebert (1980). Estos datos fueron promediados y relacionados con las medias de infección observadas en campo para cada variedad mediante el coeficiente de correlación de Pearson y regresión lineal simple.

\section{RESULTADOS}

En la primera etapa de evaluación de la Capacidad de infección a campo, para la variedad Caninde 1 , la media estuvo entre 0 y 0,90 , mientras que en la segunda etapa la media fue de entre $0 \mathrm{y}$ 1,20 (Tabla 1). La mayor media de infección, en ambas evaluaciones correspondió al cultivo inoculado con suspensiones preparadas al momento, es decir, con cero días de conservación.

El tratamiento 1, que tuvo cero días de conservación, fue estadísticamente diferente a los demás tratamientos en estudio en ambas evaluaciones. Mientras que, las suspensiones conservadas por $8, \quad 15,24$ y 30 días, respectivamente, fueron estadísticamente similares en cuanto a la capacidad de infección.

Para la variedad Caninde 11, en la primera evaluación, las medias de la capacidad de infección estuvieron entre 0 y 1,0. Las espigas inoculadas con suspensiones con cero días de conservación presentaron el mayor índice de infección, mientras que las inoculadas con suspensiones con 24 y 30 días de conservación, respectivamente, presentaron las medias más bajas.

En la segunda evaluación, los valores de medias de índice de infección para la variedad Caninde 11 estuvieron entre 0 y 3,0. Las espigas inoculadas con suspensiones preparadas al momento de la inoculación fueron las que presentaron mayor índice de infección, siendo este tratamiento significativamente diferente a los demás. 
Tabla 1. Medias de infección de $P$. oryzae, para Caninde 1 y Caninde 11 a los 15 y 30 días de la inoculación en ensayo de campo.

\begin{tabular}{ccccccccc}
\hline & \multicolumn{4}{c}{ Caninde 1 } & \multicolumn{4}{c}{ Caninde 11 } \\
\hline Tratamiento & $\begin{array}{c}\text { Media a los } \\
\text { 15 ddi }\end{array}$ & $\begin{array}{c}\text { Media a los } \\
\text { 30 ddi }\end{array}$ & $\begin{array}{c}\text { Media a los } \\
\text { 15 ddi }\end{array}$ & $\begin{array}{c}\text { Media a los } \\
\text { 30 ddi }\end{array}$ \\
\hline T1 & 0,90 & b & 1,20 & b & 1,00 & b & 3,00 & b \\
\hline T2 & 0,10 & a & 0,20 & a & 0,20 & a & 0,20 & a \\
\hline T3 & 0,10 & a & 0,00 & a & 0,30 & a & 0,30 & a \\
\hline T4 & 0,00 & a & 0,00 & a & 0,00 & a & 0,20 & a \\
T5 & 0,00 & a & 0,00 & a & 0,00 & a & 0,00 & a \\
\hline
\end{tabular}

T1: 0 días de conservación, T2: 8 días de conservación, T3: 15 días de conservación, T4: 24 días de conservación, T5: 30 días de conservación. Medias con una letra en común no son significativamente diferentes $(p>0.05)$. Diferencias establecidas mediante la comparación de medianas utilizando la prueba de Kruskall-Wallis.

Las reacciones presentadas por las variedades a la inoculación a campo se detallan en la Tabla 2, observándose que el nivel de resistencia de las variedades inoculadas con la suspensión de cero días de conservación (tratamiento 1), se redujo en la evaluación realizada a los 30 días, pasando, la variedad Caninde 1, de resistente a moderadamente resistente. Mientras, la variedad Caninde 11 pasó de moderadamente resistente a susceptible. En los demás tratamientos, el nivel de resistencia se mantuvo en ambos periodos de evaluación.

En las evaluaciones efectuadas en condiciones de invernadero la media de infección para la variedad Caninde 1 fue de cero, no presentando diferencias significativas entre los tratamientos empleados, en tanto que la variedad Caninde 11 presentó un índice de infección con media de 2,48 en el tratamiento 1 , siendo este índice igual a cero para los demás tratamientos (Tabla 3 ).

La clasificación de las variedades al ser inoculadas en invernadero y evaluadas a los 15 días de la inoculación se puede apreciar en la Tabla 4. La variedad Caninde 1 demostró resistencia a la cepa P14-038 de $P$. oryzae, mientras que la variedad Caninde 11 expresó ser moderadamente susceptible a la cepa 038 con cero días de conservación, con los demás periodos de conservación, ambas variedades fueron resistentes.

En cuanto a la relación de las medias de infección y la cantidad de esporas sin germinar en las suspensiones con distintos periodos de conservación (Figura 1), en la variedad

Tabla 2. Clasificación de las variedades según su reacción frente a los diferentes tiempos de conservación de cepas inoculadas.

\begin{tabular}{cccccc}
\hline & & \multicolumn{3}{c}{ Clasificación según tipo de reacción en campo } \\
\cline { 3 - 6 } Tratamientos & \multicolumn{2}{c}{ Caninde 1 } & \multicolumn{2}{c}{ Caninde 11 } \\
\hline- & & 15 días & 30 días & 15 días & 30 días \\
\hline & T1 & $\mathrm{R}$ & MR & MR & $\mathrm{S}$ \\
& T2 & $\mathrm{R}$ & $\mathrm{R}$ & $\mathrm{R}$ & $\mathrm{R}$ \\
& $\mathbf{T 3}$ & $\mathrm{R}$ & $\mathrm{R}$ & $\mathrm{R}$ & $\mathrm{R}$ \\
& $\mathrm{T4}$ & $\mathrm{R}$ & $\mathrm{R}$ & $\mathrm{R}$ & $\mathrm{R}$ \\
& $\mathrm{T5}$ & $\mathrm{R}$ & $\mathrm{R}$ & $\mathrm{R}$ & $\mathrm{R}$ \\
\hline
\end{tabular}

T1: 0 días de conservación, T2: 8 días de conservación, T3: 15 días de conservación, T4: 24 días de conservación, T5: 30 días de conservación. R: Resistente, MR: moderadamente resistente, MS: moderadamente susceptible, S: Susceptible. 
Caninde 1, el coeficiente de correlación fue de 0,98, mientras que en la variedad Caninde 11 fue 0,97; estos valores indican una fuerte correlación entre las variables. La regresión lineal simple

Tabla 3. Medias de infección de $P$. oryzae, para las variedades Caninde1 y Caninde 11, a los 15 días de la inoculación en ensayo de invernadero.

\begin{tabular}{ccc}
\hline Tratamiento & $\begin{array}{c}\text { Media a los 15 ddi } \\
\text { Caninde 1 }\end{array}$ & $\begin{array}{c}\text { Media a los 15 ddi } \\
\text { Caninde 11 }\end{array}$ \\
\hline $\boldsymbol{T 1}$ & $0,00 \mathrm{a}^{*}$ & $2,48 \mathrm{~b}$ \\
$\boldsymbol{T 2}$ & $0,00 \mathrm{a}$ & $0,00 \mathrm{a}$ \\
$\boldsymbol{T 3}$ & $0,00 \mathrm{a}$ & $0,00 \mathrm{a}$ \\
$\mathbf{T 4}$ & $0,00 \mathrm{a}$ & $0,00 \mathrm{a}$ \\
$\boldsymbol{T 5}$ & $0,00 \mathrm{a}$ & $0,00 \mathrm{a}$ \\
\hline
\end{tabular}

Medias con una letra en común no son significativamente diferentes $(p>0.05)$.

Tabla 4. Clasificación de las variedades según su reacción frente a las suspensiones con diferentes tiempos de conservación en ensayo de invernadero.

\section{Clasificación según tipo de reacción}

\begin{tabular}{ccc}
\cline { 2 - 3 } Tratamiento & Caninde 1 & Caninde 11 \\
\hline T1 & $\mathrm{R}$ & $\mathrm{MS}$ \\
T2 & $\mathrm{R}$ & $\mathrm{R}$ \\
T3 & $\mathrm{R}$ & $\mathrm{R}$ \\
T4 & $\mathrm{R}$ & $\mathrm{R}$ \\
T5 & $\mathrm{R}$ & $\mathrm{R}$ \\
\hline
\end{tabular}

R: Resistente, MR: moderadamente resistente, MS: moderadamente susceptible, S: Susceptible

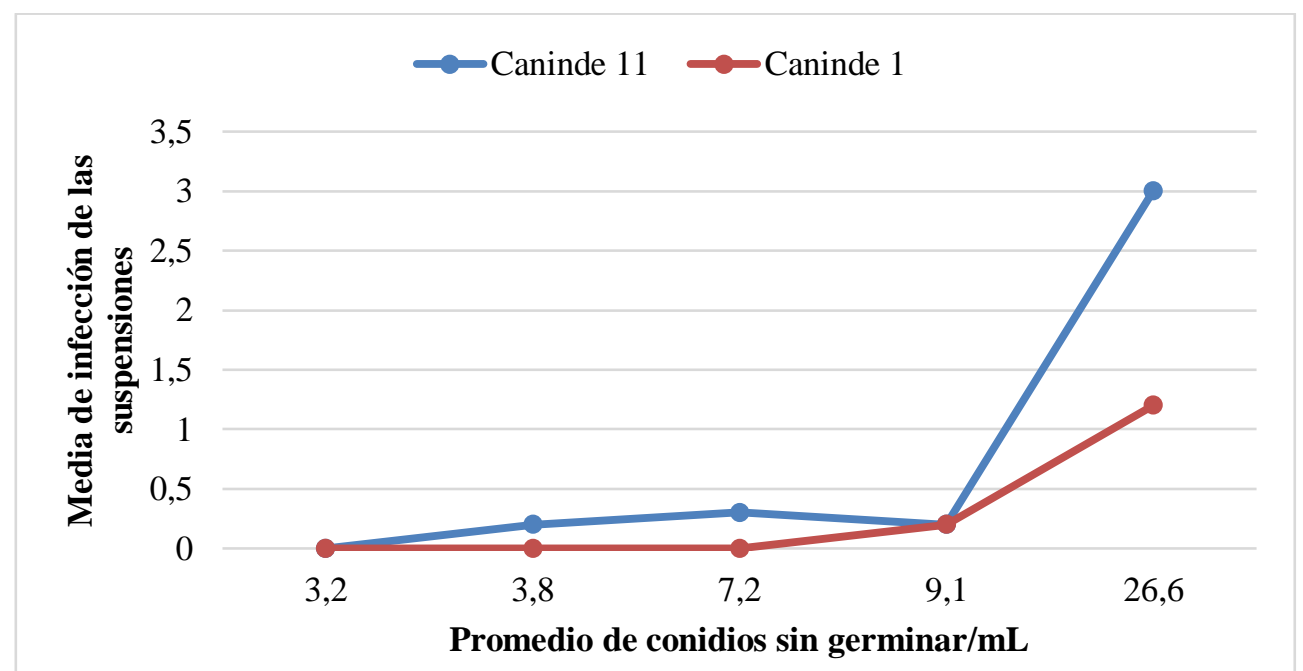

Figura 1. Relación entre la media de infección y la cantidad de conidios sin germinar en las suspensiones con diferentes periodos de conservación para las variedades Caninde 1 y Caninde 11. 


\section{DISCUSIÓN}

Las reacciones de infección presentadas por las variedades Caninde 1 y Caninde 11 en este trabajo concuerdan con los presentados previamente por Chávez \& Mohan-Kohli (2018), quienes estudiaron la reacción de ambas variedades con inóculos de misma concentración preparados al momento de la inoculación.

Guillén (2018) obtuvo datos similares estudiando la patogenicidad de cepas de Pyricularia sp. aisladas de hospederos alternativos sobre trigo con diferentes cepas, donde demostró que la capacidad de infección, representada por la media del tipo de reacción de las variedades, varió entre 0 y 0,80 para la variedad Caninde 1 ; y entre 0 y 2,40 para la variedad Caninde 11. Dentro del mismo estudio las variedades inoculadas tuvieron el comportamiento esperado, actuando como resistente (Caninde 1) y susceptible (Caninde 11), en ambas épocas frente a las distintas cepas inoculadas.

En el ensayo a campo la media mensual de temperaturas máximas para los meses de junio y julio fueron de $21,9^{\circ} \mathrm{C}$ y $21,2^{\circ} \mathrm{C}$, respectivamente; mientras que la precipitación registrada fue de 56,0 mm y $57 \mathrm{~mm}$ para los meses de junio y julio, respectivamente mientras que la temperatura $\mathrm{y}$ humedad ideales para el establecimiento del patógeno son de $28^{\circ} \mathrm{C}$ y humedad superior al $90 \%$ (Santos \& Campelo, 1977; Bedendo \& Prabhu, 2005; Fernandez \& Pavan, 2010). Las condiciones ambientales pudieron influir en la capacidad de infección de $P$. oryzae, ya que se observó un bajo nivel de infección principalmente para la variedad Caninde 11, considerada susceptible.

Las infecciones realizadas a campo e invernadero fueron llevadas a cabo con suspensiones de esporas, estas son estructuras especializadas para la reproducción asexual del hongo. Las esporas tienen las características que bajo ciertas condiciones ambientales que involucran luz, temperatura, humedad $\mathrm{y}$ disponibilidad de nutrientes pueden germinar iniciando así su activación y desarrollo (Regueira, 2017).

Los hongos fitopatógenos como Pyricularia oryzae, emplean esporas como mecanismo de infección, en donde cada etapa del ciclo de infección se encuentra regulado por factores bióticos y abióticos. Estas etapas incluyen: adhesión, germinación, formación de apresorio, penetración e invasión (Cruz et al., 2016).

En un estudio realizado por Nogueira (2009) se ha propuesto un modelo de infección de Pyricularia grisea en donde se estableció que después de la adhesión de la espora a la superficie del hospedero, la germinación puede llevarse a cabo por un periodo de 0 a 2 horas permaneciendo esta viable y activa para la formación del apresorio. Al ser la germinación un proceso que se activa por la influencia de condiciones ambientales es posible que las condiciones de almacenamiento de las suspensiones realizadas para este ensayo hayan favorecido la germinación de esporas.

Se ha observado que la capacidad de infección disminuye al aumentar el tiempo de conservación, y el número de esporas germinadas. A pesar de 
mantener en restricción factores como nutrientes y temperatura optima, las células se adaptan progresivamente a la condición. Las esporas pregerminadas en las suspensiones presentan pérdida de capacidad para realizar la infección cuando entran en contacto con los hospederos (Caninde1 y Caninde11).

Es necesario realizar análisis bioquímicos para determinar la causa especifica de este efecto, pero teniendo en cuenta el ciclo de infección del patógeno es muy probable que esta pérdida pueda deberse a que una vez germinadas en las suspensiones, las esporas no pueden desarrollar el apresorio, una de las estructuras indispensables involucradas en el proceso de invasión y desarrollo de enfermedad. El apresorio permite la entrada del hongo a las células vegetales permitiendo la captación de nutrientes y su crecimiento.

Se hace necesario continuar con los ensayos de efectividad de conservación de inóculos bajo condiciones más restringidas que no favorezcan la activación de las esporas. Así como la conservación de las suspensiones por periodos más cortos de tiempo que permitan estimar la viabilidad de las esporas pregerminadas (Alarcón, 2006).

Declaración de interés. Los autores declaran no tener conflicto de intereses.

\section{REFERENCIAS BIBLIOGRÁFICAS}

Alarcón, D. I. (2006). Evaluación de técnicas de conservación para hongos filamentosos y levaduriformes en el cepario de la pontificia Universidad Javeriana. (Tesis de grado).Bogotá, Colombia. Pontificia Universidad Javeriana

Bedendo, J. F. \& Prabhu, A.S. (2005). Doenças do arroz (Oryza sativa). Em H. Kimati, L. Amorim, J. Rezende, A. Bergamin, L. Camargo. Manual de Fitopatologia: doenças das plantas cultivadas. Vol. 2. (4 ed.), pp. 79-
90. São Paulo, BR. Ceres.

CAPECO (2021). Estadísticas. Disponible en: http//capeco.org.py/

Chávez, A. R. \& Mohan-Kohli, M. (2018). Patogenicidad de Magnaporthe oryzae en variedades y líneas de trigo cultivadas en Paraguay. Revista mexicana de fitopatología, 36 (2), 276-286.

Chávez, A., Reyes, M. \& Kohli, M. (2020). Evaluación de la resistencia de genotipos de trigo a la infección de Magnaporthe oryzae en condiciones de campo. En Instituto paraguayo de tecnología agraria (IPTA), Cámara de exportadores y comercializadores de cereales y oleaginosas (CAPECO), Instituto de biotecnología agrícola (INBIO) (eds). Resultados de la investigación del cultivo de trigo ciclo 2019, pp. 95-97.

Cruz, M. F. A., Rios, J. A., Araujo, L. \& Rodrigues, F. A. (2016). Infection process of Pyricularia oryzae on the leaves of wheat seedlings. Tropical Plant Pathology, 41 (2), 123-127.

Fernandez, J. \& Pavan, W. (2010). Sistemas de predicción para enfermedades em cereales de inverno: fusariosis y brusone. In Embrapa Trigo-Artigo em anais de congresso (ALICE). In S. Pereyra, M. D. de Ackermann, S. Germán \& K. Cabrera, K. Manejo de enfermedades en trigo y cebada. Montevideo. INIA. p. 37-39. (INIA. Serie técnica, 189).

French, E. R. \& Hebert, T. T. (1980). Métodos de investigación fitopatológica. San José, Costa Rica. Instituto Interamericano de Ciencias Agrícolas. Bib. Orton IICA/CATIE.

Guillén, A. M. (2018). Patogenicidad de cepas de Pyricularia sp. Aisladas de hospederos alternativos sobre trigo (Tesis de grado). Caacupé, Paraguay. Universidad Católica Nuestra Señora de la Asunción.

Kohli, M. M., Mehta, Y. R., Guzman, E., Viedma, L. \& Cubilla L. E. (2011). Pyricularia: una amenaza para el cultivo del trigo. Plant Breed. 47, 130-134.

Marangoni, M. S., Nunes, M. P., Fonseca, N. \& Mehta, Y. R. (2013). Pyricularia blast on white oats: a new threat to wheat cultivation. Tropical plant pathology, 38 (3), 198202.

Nogueira, L. (2009). Estructura Poblacional y Análisis Epidemiológicos de Pyricuaria grisea. (Tesis de Maestria en Fitopatologia). Universidad de Brasilia, Brasil.

Regueira Paz, N. (2017). Detección e identificación de hongos fitopatógenos y antagonistas en muestras de compost. (Tesis de Maestría en Biotecnología Avanzada). Universidad de Coruña, España.

Santos, A. A. \& Campelo, G. D. A. (1977). Doenças do arroz (Oryza sativa L.) no Estado do Piauí e seu controle. EMBRAPA, UEPAE de Teresina.

Tagle, A. G., Chuma, I. \& Tosa, Y. (2014). Rmg7, a New Gene for Resistance to Triticum Isolates of Pyricularia oryzae Identified in Tetraploid Wheat. Genetics and resistance, 105 (4), 495-499.

Valent, B., Farrall, L. \& Chumley, F. G. (1991). Magnaporthe grisea genes for pathogenicity and virulence identified through a series of backcrosses. Genetics, 127 (1), 87101. 